\title{
A Rare Case Report of Unilateral Involvement of Basal Ganglia and Thalamus in a Case of Hypoxic Brain Injury
}

\author{
Article by Sunil chowdary Minnekanti, Monika sai paida, Sushant Duddala, Nerin \\ Duddala, Snigdha shanti, Varsha Pulijal \\ Email:-crazychou@gmail.com
}

\begin{abstract}
Hypoxic injury of brain can occur at any age from an infant to an adult, also called as global hypoxic ischaemic injury. The patients usually presents with a history of asphyxiation, drowning, cardiac arrest or respiratory arrest. Basalganglia, thalami, cerebral cortex (the sensorimotor and visual cortices are predominantly involved, though involvement is often diffuse), cerebellum, hippocampi are the areas usually affected in the brain. Usually the involvement is bilateral in deep nuclei we here present a rare case of unilateral involvement of basal ganglia in a case of hypoxic brain injury.
\end{abstract}

\section{Introduction}

- Hypoxic-ischaemic cerebral injury occurs at any age group, though the aetiology is significantly different in infants, children and adults.

- Older children: Drowning and asphyxiation are the most common causes of.

- Adults: Due to result of cardiac arrest or cerebrovascular disease, with secondary hypoxemia/hypoperfusion.

- The basal ganglia and thalamus are paired deep gray matter structures that may be involved by a wide variety of disease entities. The basal ganglia are highly metabolically active and are symmetrically affected in toxic poisoning, metabolic abnormalities, and neurodegenerative disorders with brain iron accumulation. Both the basal ganglia and thalamus may be affected by other systemic or metabolic disease, degenerative disease, and vascular conditions. Radiologists may detect bilateral abnormalities of the basal ganglia and thalamus in different acute and chronic clinical situations, and although magnetic resonance (MR) imaging is the modality of choice for evaluation, the correct diagnosis can be made only by taking all relevant clinical and laboratory information into account.

- The neuroimaging diagnosis is influenced not only by detection of specific MR imaging features such as diffusion restriction and the presence of hemorrhage, but also by detection of abnormalities involving other parts of the brain, especially the cerebral cortex, brainstem and white matter.

- During the acute period after diffuse cerebral anoxia, the results of conventional MR imaging and CT scanning may be normal, or the images and scans may show only subtle abnormalities. The imaging findings of diffuse cerebral anoxia include obscured grey-white matter junctions, abnormal appearance of deep grey matter nuclei, infarctions in regions between major arterial territories, and laminarnecrosis.

- Because of its poor prognosis, a method that rapidly helps in diagnosis of diffuse cerebral anoxia is required. PET and SPECT may be helpful in the diagnosis of diffuse cerebral anoxia, but may be difficult to perform in hypoxic brain damage group patients. The use of diffusion-weighted MR imaging for the diagnosis of acute cerebral infarction has been described, but this imaging technique has not been used to examine patients with diffuse cerebral anoxia ${ }^{(3)}$. 


\section{Case report}

- 24 year old male alleged case of poisoning and attempted hanging after poisoning came with history of unconsciousness. On examination there is no history of protrusion of tongue or convulsions or any signs of head injury. The patient was referred from outside hospital for further management. On admission the patient was intubated and is on ventilator support. On examination the patient was sedated, pupils dilated $(3 \mathrm{~mm})$ sluggishly reactive with minimal eye opening and moving all four limbs. The patient is tachycardic, blood pressure was 110/70 mm $\mathrm{Hg}$ and febrile. There was one episode of GTCS after the admission. Based on the history of poisoning and hanging, routine toxicology screen and drug screening were performed. The labs revealed high amounts of cholinesterase of $6244.7 \mu / 1$ $(2700-4100)$. The routine drug screen was negative. Serum $\mathrm{K}^{+}$was 5.7.

On admission $\mathrm{ABG}$ revealed the following findings

- $\mathrm{pH}$ of $7.4(7.35-7.45)$

- $\mathrm{PCo} 227(35-45) \mathrm{mm} \mathrm{Hg}$.

- PO2 250 (83- 105$) \mathrm{mm} \mathrm{Hg}$.

- $\mathrm{Hco3}^{-} 18.2 \mathrm{mmol} / \mathrm{lt}$

- $\mathrm{K}^{+}-3.7(3.5-5) \mathrm{mmol} / \mathrm{lt}$

- $\mathrm{Na}^{+}-133(135-146) \mathrm{mmol} / \mathrm{lt}$.

- $\mathrm{Ca}^{+2}-1.06(1.15-1.29) \mathrm{mmol} / \mathrm{lt}$.

- $\mathrm{Cl}^{-}-110 \mu / 1$.

- Anion gap was $4.8 \mathrm{mmol}$.

- Renal function tests and liver function tests are normal.

- Echo showed mild grade I Mitral regurgitation, aortic valve is sclerotic and is tri leaflet, mitral diastolic flow is altered, Ejection Fraction is $40 \%$ with a kinetic left ventricular apex and showed minimal pericardial effusion. Usg abdomen and pelvis revealed no significant abnormality. $\mathrm{Ct}$ scan of brain performed on the day of admission did not reveal any abnormality.

- Mri brain was performed 3 days after admission in our institute as the patient is not showing improvement, which revealed the following findings.

- Scattered areas of abnormal hyperintense signal on T2-weighted and FLAIR sequences in right occipital region, right temporo-occipital region, right basal ganglia, right thalamus, right high frontoparietal region and left high frontoparietal region and these areas showed diffusion restriction.

- The signal abnormality also involved bilateral peri-Rolandic cortical regions with predominant involvement of the cortex with involvement of the subcortical white matter in right occipital regional. The left basal ganglia and left thalamus appear normal.

- The rest of the brain parenchyma, cerebellum and brainstem does not reveal any abnormality. 


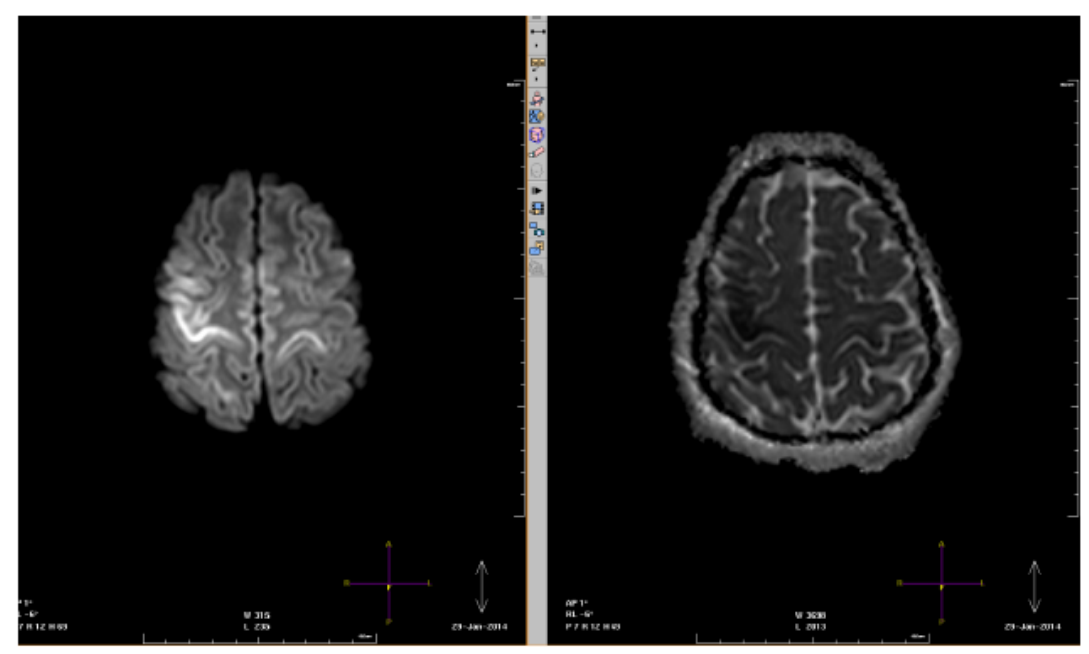

DWI images show area of diffusion restriction in bilateral high frontoparietal region with corresponding hypointensity on ADC maps.

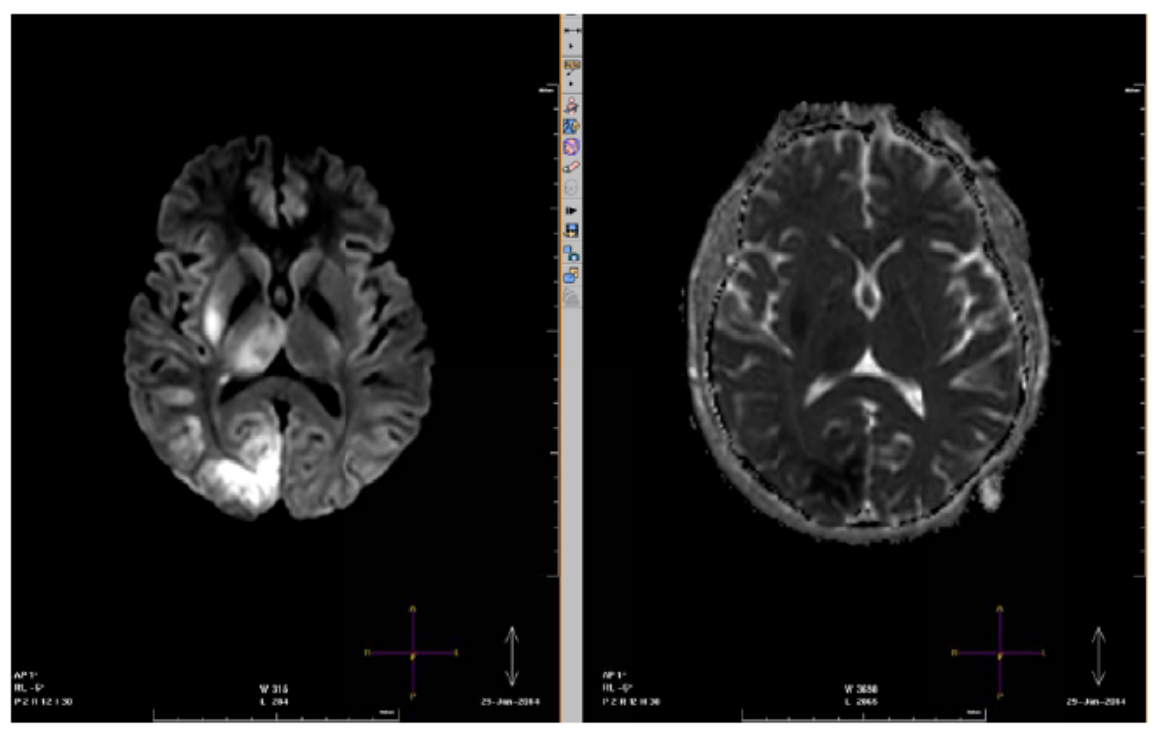

DWI images show area of diffusion restriction in right basal ganglia and right thalamus and right posterior occipital lobe with corresponding ADC maps. 
South American Journal of Clinical Research

Special Edition 2016

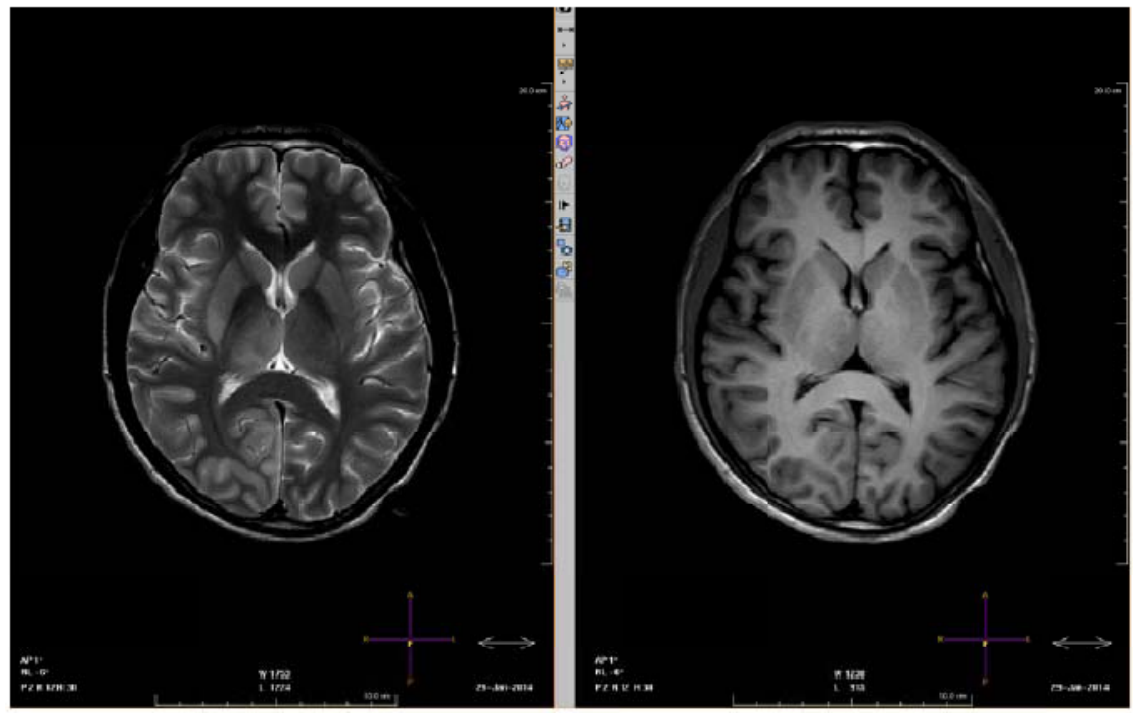

T1 weighted images show minimal hypointensity in the areas of corresponding diffusion restriction and minimal hyperintensity on $\mathrm{T} 2$ weighted images.

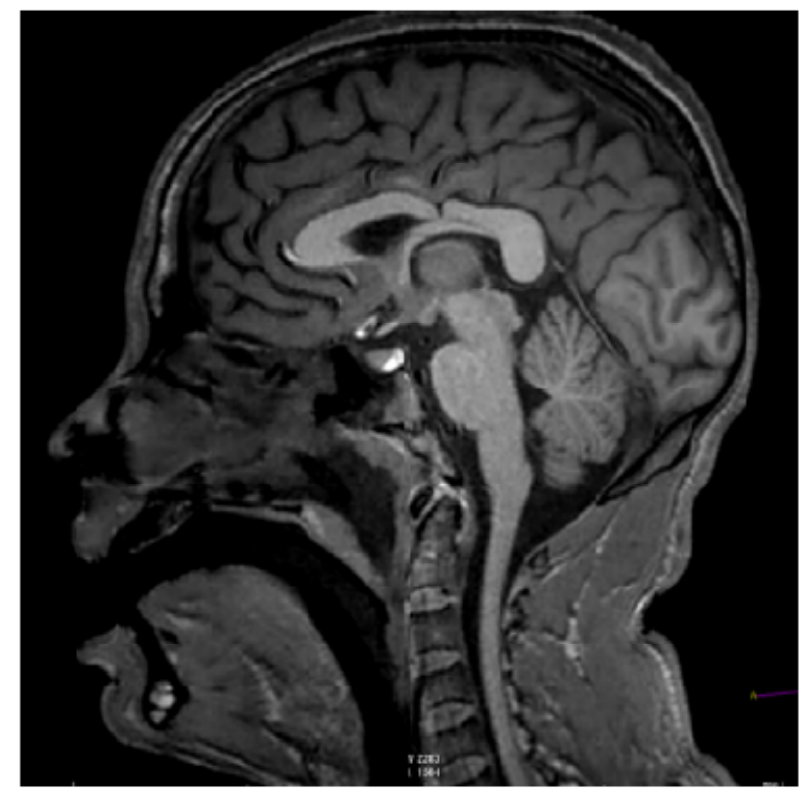

T1 weighted images show mild vacuity in the continuity of the body of the right corpus callosum which is confirmed on the DTI imaging showing less "fa" value. 
South American Journal of Clinical Research Special Edition 2016
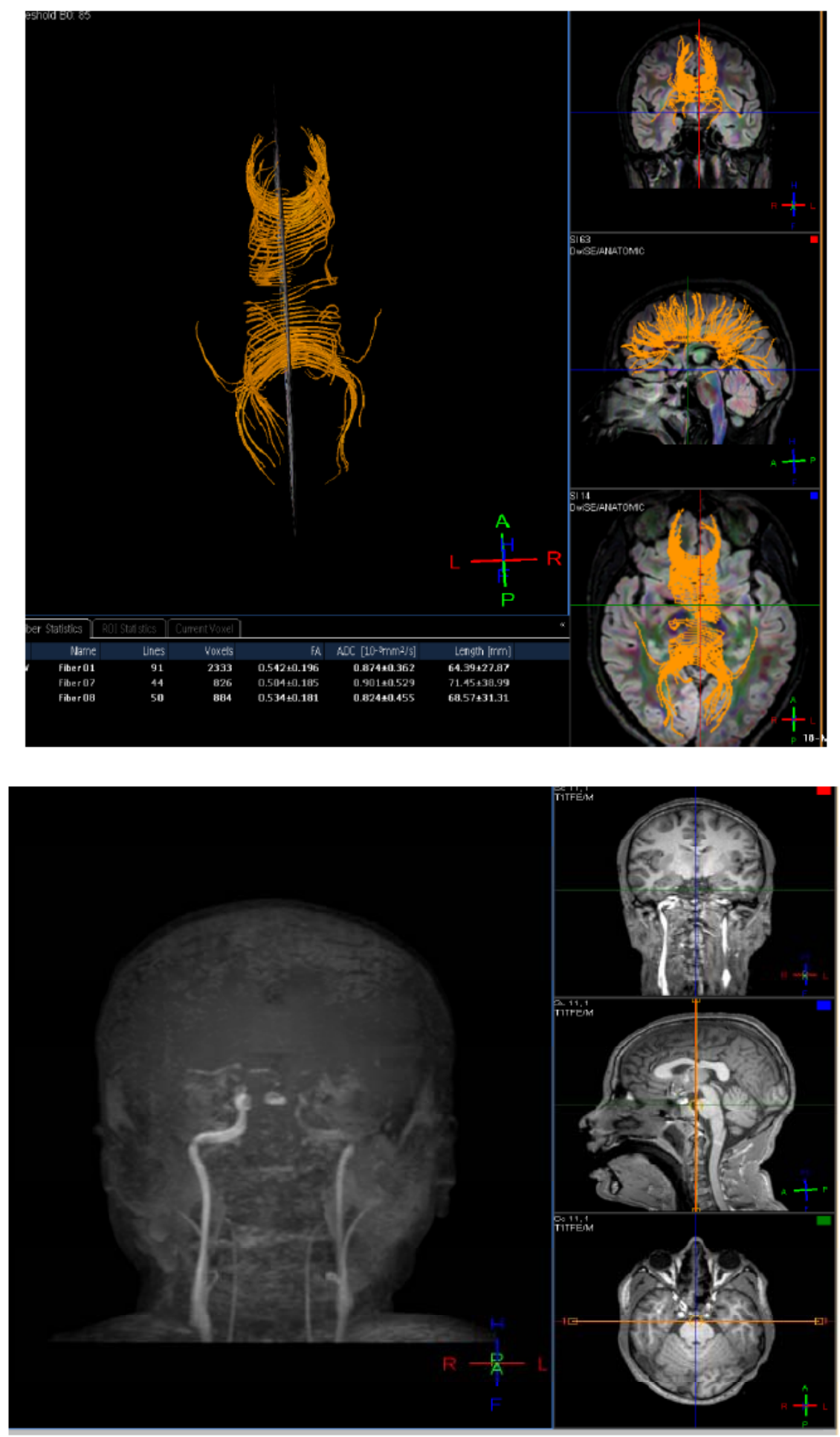
South American Journal of Clinical Research

Special Edition 2016

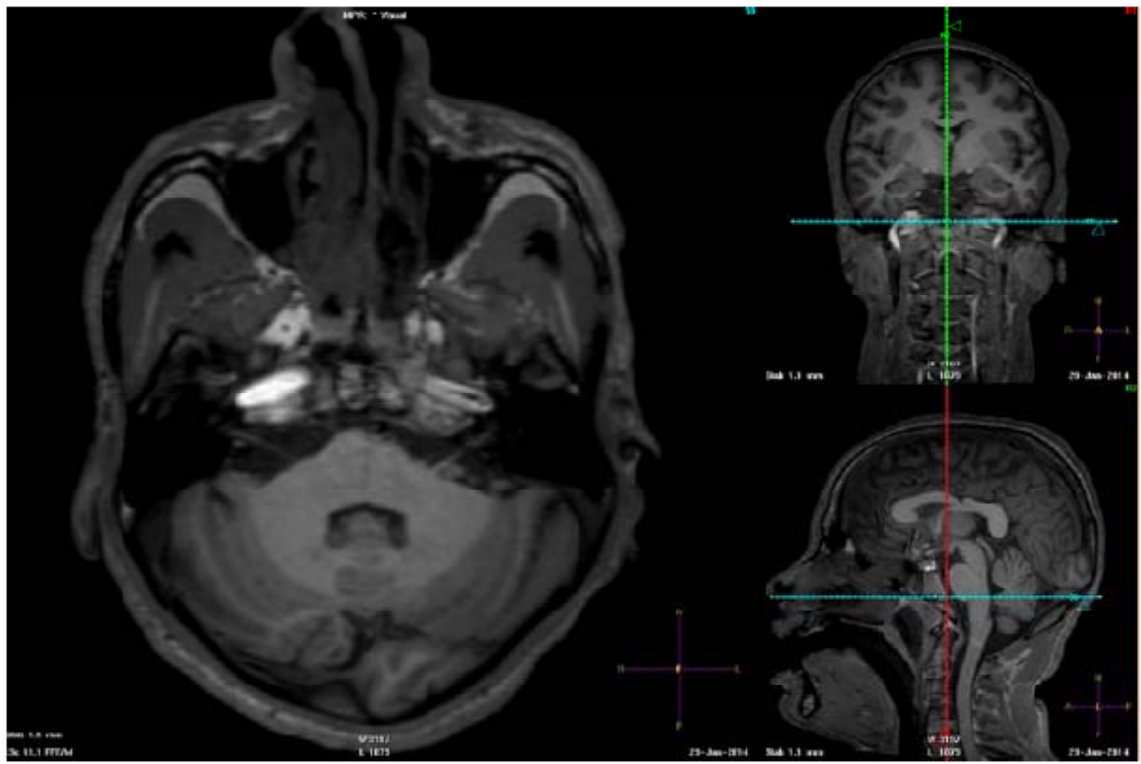

- Non contrast 2D-TOF angiography revealed non occlusion of both vertebral arteries and no dissection of bilateral ICA's.

\section{Discussion}

Regardless of the cause of injury, the common underlying physiopathologic processes that result in hypoxic ischaemic injury is decreased cerebral blood flow (ischemia) and reduced blood oxygenation (hypoxemia). In general, infants and children are more likely to suffer asphyxia events, which result in hypoxemia and brain hypoxia. With prolonged hypoxemia there is cardiac hypoxia which leads to diminished cardiac output which results in brain ischemia. Thus, brain injury resulting from asphyxia is the consequence of ischemia with superimposed cardiac hypoxia. In fact, acute hypoxemia without superimposed ischemia is less likely to cause injury, unless there is prolonged hypoxia. Adults more frequently suffer brain ischemia as a result of cardiac arrest or cerebrovascular disease, with secondary hypoxia due to reduced blood flow. It is well known that global hypoxic-ischemic insults do not affect all brain structures uniformly. Rather, certain tissues in the brain are more likely to be injured and are injured earlier than others, a concept known as selective vulnerability.

The patterns of observed injury reflect dysfunction of selected excitatory neuronal circuits, which causes a complex cascade of unwanted biochemical events and, ultimately, selective neuronal death. Brain ischemia causes a change of metabolism from oxidative phosphorylation to anaerobic metabolism, which is highly inefficient. This change causes rapid depletion of adenosine triphosphate (ATP) and causes lactate accumulation within cells, and eventual loss of normal cellular membrane function. Depolarization of presynaptic neuronal cell membranes causes a massive release of excitatory neurotransmitters in particular, glutamate. In immature brains, glutamate binds predominantly to $N$-methyld- aspartate (NMDA) receptor-mediated calcium $\left(\mathrm{Ca}^{+2}\right)$ channels. Activation of NMDA receptors results in an influx of $\mathrm{Ca}^{+2}$ into postsynaptic neurons, which triggers a number of cytotoxic processes, including activation of membrane phospholipases and production of the oxygen free radicals (such as nitric oxide) that damage cell membranes and internal constituents. Damage to mitochondria may ensure, causing further loss of ATP production and, ultimately energy depletion. Severe energy depletion results in rapid cell death from necrosis. With lesser degrees of energy depletion, neurons may survive the initial insult, only to undergo a delayed form 
of programmed cell death known as apoptosis. Apoptosis appears to play a significant role in injury to the immature brain.

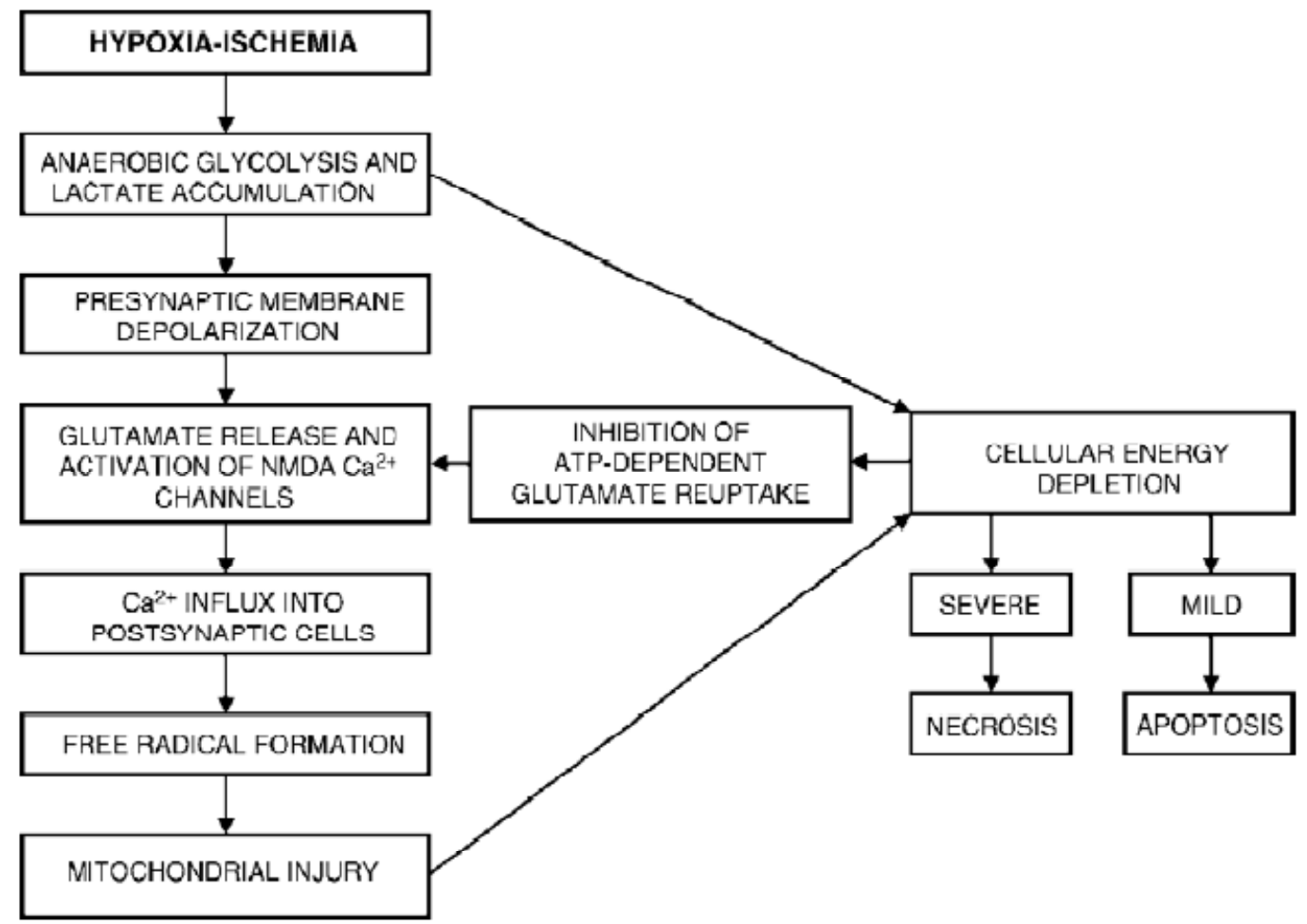

Adopted from Benjamin Y. Huang, MD, MPH •Mauricio Castillo, MD Hypoxic-

\section{Ischemic}

Brain Injury: Imaging Findings from Birth to Adulthood

From the model just described, we can draw the following conclusions:

- The areas of the brain with the highest concentrations of glutamate or other excitatory amino acid receptors are more susceptible to excite toxic injury that occurs as a result of hypoxiaischemia.

- The areas of the brain with the greatest energy demands become energy depleted most rapidly during hypoxic ischemia, and are therefore injured early on.

- Because of delayed cell death from apoptosis, some injuries may not be evident until days after the initial insult has occurred.

- These factors help to explain the relatively specific patterns of injury that can be observed in patients with Hypoxic ischaemic injury. In any given patient, the sites in the brain that tend to be most vulnerable to hypoxic injury will be determined largely by the maturity of the brain, which is a function of patient age and, in infants, gestational age at birth.

- This is why Hypoxic ischaemic injury in the perinatal period differs from Hypoxic ischaemic injury in adults or even in older infants and the imaging appearance of Hypoxic ischaemic injury differs between term and preterm neonates ${ }^{(3)}$.

- One must be aware of the degree of brain maturity at the time of the insult when interpreting studies for suspected Hypoxic ischaemic injury.

- The severity of a hypoxic ischemic insult also plays an important role in determining the distribution of injuries in the brain.

- Episodes of severe hypoxia-ischemia result in a different injury pattern compared with less severe insults.

- Duration of insult also seems to be a key determinant of the pattern of injury in HII, since insults of short duration usually do not result in brain injury. 
- It has been suggested that, in the paediatric population, an arrest must typically last at least 15 minutes for brain injury to occur.

\section{Blood supply to the basal ganglia and thalamus}

- Posterior Inferior Cerebellar Artery (PICA in blue).

The PICA territory is on the inferior occipital surface of the cerebellum and is in equilibrium with the territory of the AICA in purple, which is on the lateral side. The larger the PICA territory, the smaller the AICA and viceversa ${ }^{(2,4)}$.

- Superior Cerebellar Artery (SCA in grey).

The SCA territory is in the superior and tentorial surface of the cerebellum.

- Branches from vertebral and basilar artery.

These branches supply the medulla oblongata (in blue)and the pons (in green).

- Anterior Choroideal artery (AchA in blue).

The territory of the Ach artery is part of the hippocampus, the posterior limb of the internal capsule and extends upwards to an area lateral to the posterior part of the cella media.

- Lenticulo-striate arteries.

The lateral LSA's (in orange)are deep penetrating arteries of the middle cerebral artery (MCA).Their territory includes most of the basal ganglia.

The medial LSA's (indicated in dark red) arise from the anterior cerebral artery (usually the A1-segment).

Heubner's artery is the largest of the medial lenticulostriate arteries and supplies the anteromedial part of the head of the caudate and anteroinferior internal capsule.

- Anterior cerebral artery (ACA in red).

The ACA supplies the medial part of the frontal and the parietal lobe and the anterior portion of the corpus callosum, basal ganglia and internal capsule.

- Middle cerebral artery (MCA in yellow).

The cortical branches of the MCA supply the lateral surface of the hemisphere, except for the medial part of the frontal and the parietal lobe (anterior cerebral artery), and the inferior part of the temporal lobe (posterior cerebral artery).

The deep penetrating LSA-branches are discussed above.

- Posterior cerebral artery (PCA in green).

P1 extends from origin of the PCA to the posterior communicating artery, contributing to the circle of Willis.

Posterior thalamoperforating arteries branch off the P1 segment and supply blood to the midbrain and thalamus.

Cortical branches of the PCA supply the inferomedial part of the temporal lobe, occipital pole, visual cortex, and splenium of the corpus callosum. 


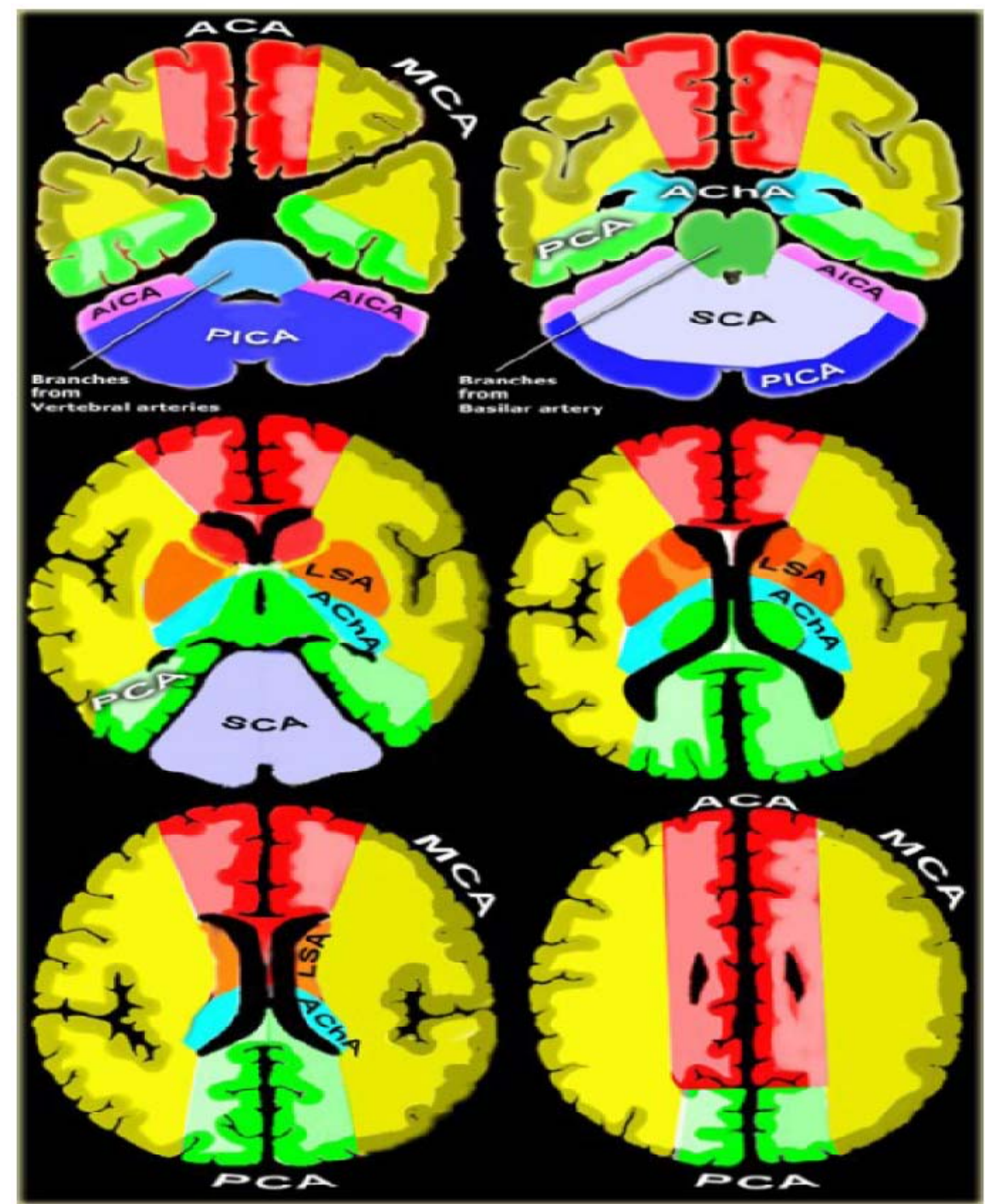

Diagrammatic representation of the blood supply of human brain parenchyma

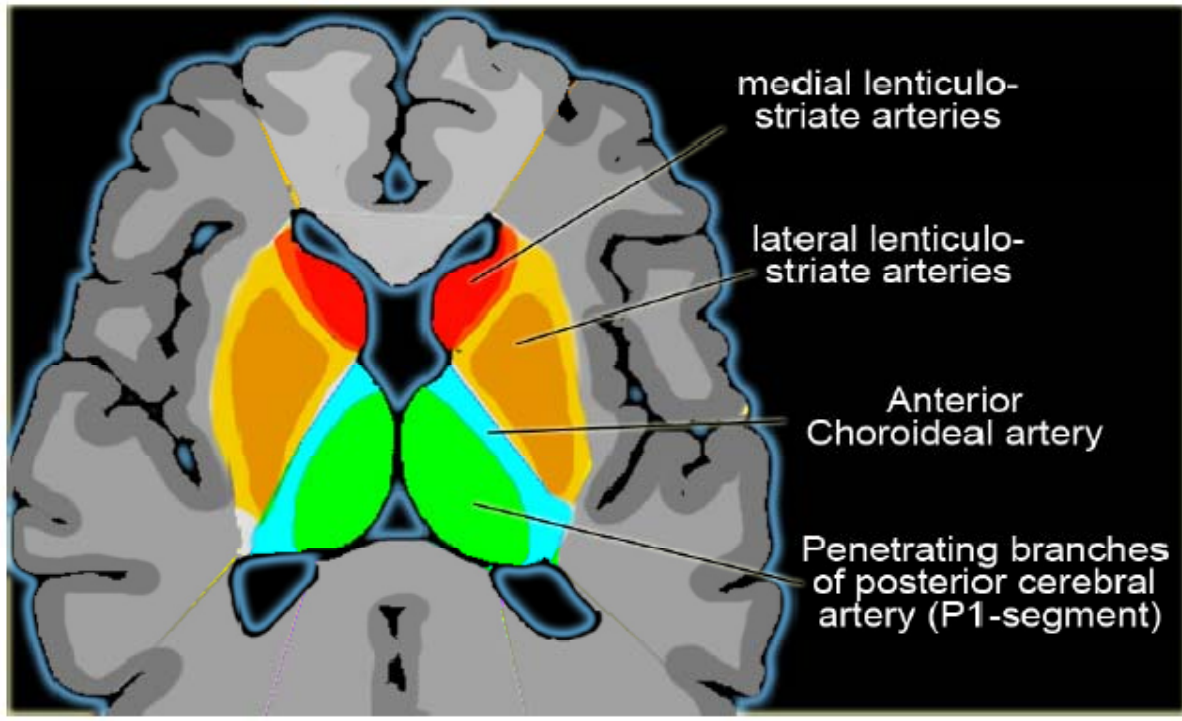

In our case scenario the possibility of unilateral involvement of the basal ganglia and thalamus might be due to the cause of unilateral compression of the lateral lenticulo- 
South American Journal of Clinical Research

Special Edition 2016

striate branches and the penetrating branches of posterior cerebral artery (P1 segment) on left side during the alleged incident of hanging which spared the left basal ganglia and thalamus. ${ }^{(2,4)}$

\section{Conclusion}

Hypoxic ischaemic injury can be occurred in both children and adults due to various causes MR imaging plays a crucial role in evaluating subtle changes of hypoxic brain injury. Though there is overlap of findings on MRI a clinical discussion and observing the clinical details, history and symptoms help the radiologist in narrowing down the possible diagnosis of the cause of hypoxia. MR angiogram or CT angiogram may be necessary to identify the dissection in vertebral arteries in a case of hanging.

\section{Bibilography}

1. Arbelaez, a, Castillo, M., \& Mukherji, S. K. (1999). Diffusion-weighted MR imaging of global cerebral anoxia. AJNR. American Journal of Neuroradiology, 20(6), 999-1007. Retrieved from http://www.ncbi.nlm.nih.gov/pubmed/10445435

2. Huang, B. Y. (2008). Brain Injury: Imaging OBJECTIVES. RadioGraphics, 28(2), 417-439. http://doi.org/10.1148/rg.282075066

3. Renard, D., Castelnovo, G., Campello, C., Bouly, S., Floch, A. Le, Thouvenot, E., ...Taieb, G. (2014). Thalamic Lesions: A Radiological Review, 2014.

4. The vascular territories in the cerebellum and brainstem: CT and MR study. by Savoiardo M, Bracchi M, Passerini A, Visciani A. AJNR Am J Neuroradiol. 1987 Mar-Apr;8(2):1 99-209. 